\title{
Civilisations
}

Revue internationale d'anthropologie et de sciences

humaines

42-1 | 1993

Amérique latine-Europe

\section{L'Amérique latine dans un monde en transition}

\section{Alberto Van Klaveren}

URL : http://journals.openedition.org/civilisations/2103

DOI : 10.4000/civilisations.2103

ISSN : 2032-0442

\section{Éditeur}

Institut de sociologie de l'Université Libre de Bruxelles

\section{Édition imprimée}

Date de publication : 1 novembre 1993

Pagination : 143-159

ISBN : 0009-8140

ISSN : 0009-8140

\section{Référence électronique}

Alberto Van Klaveren, «L'Amérique latine dans un monde en transition», Civilisations [En ligne], 42-1 | 1993, mis en ligne le 30 novembre 1996, consulté le 19 avril 2019. URL : http:// journals.openedition.org/civilisations/2103 ; DOI : 10.4000/civilisations.2103

Ce document a été généré automatiquement le 19 avril 2019

(C) Tous droits réservés 


\title{
L'Amérique latine dans un monde en transition
}

\author{
Alberto Van Klaveren
}

L'image internationale de l'Amérique latine est l'objet d'interprétations contradictoires. D'un côté, une série d'auteurs, de personnalités et d'institutions considèrent que la région est soumise à un processus de croissante "marginalisation ", dont les caractéristiques essentielles seraient sa lourde dette extérieure, ses insuffisances sociales, aggravées manifestement durant la fameuse "décennie perdue" de 1980, sa participation décroissante dans les flux mondiaux du commerce et des capitaux, la violence interne qui affecte toujours plusieurs pays de la région, la dégradation de l'environnement, et les faiblesses de systèmes démocratiques menacés qui, bien souvent, ne sont pas parvenus à se renforcer. Quant à la participation internationale de la région, cette vision pessimiste tend à fluctuer entre le lieu commun de la permanence quasi immuable de l'hégémonie des États-Unis dans la zone et l'image désolante d'une Amérique latine «qui s'est retrouvée seule » suivant l'expression pathétique et mélodramatique qui reprend un thème central du discours prononcé par Gabriel Garcia Marquez lorsqu'il reçut le prix Nobel de littérature en 1982 ; cette interprétation est souvent reprise par des leaders latino-américains, tel l'ex-président du Costa Rica et prix Nobel de la paix 1987, Oscar Arias ${ }^{1}$. Les indicateurs de ce soi-disant isolement international de l'Amérique latine seraient la moindre attention que lui accorderaient les milieux politiques et journalistiques du monde, ou alors, sa faible participation dans les grands événements internationaux des derniers temps, selon que l'évaluation se fait dans une perspective passive - l'Amérique latine comme objet de l'attention mondiale - ou active - l'Amérique latine en tant qu'acteur international. 


\section{La reprise en Amérique latine}

2 Contrairement à la vision précédente, plusieurs auteurs, hommes politiques, organes de presse et institutions relèvent des signes plus positifs en Amérique latine. Malgré la conscience généralisée que la crise des années 80 a augmenté la pauvreté et le chômage, accru les inégalités dans la distribution des revenus, provoqué une brusque réduction des taux d'investissement et contribué à pousser la région vers une position encore plus marginale dans le commerce mondial, des tendances encourageantes apparaissent dans une série de pays. Sur le plan économique, l'Amérique latine commence à récupérer les taux de croissance qu'elle enregistrait avant les années 1980, et qui ont fait d'elle l'une des régions les plus dynamiques du monde ; mais bien souvent on oublie ce phénomène, en raison d'une certaine tendance au catastrophisme dans l'analyse des réalités latinoaméricaines. Le taux moyen de croissance économique de l'Amérique latine approchait les $3 \%$ en 1991, malgré le maintien de la récession au Brésil et, dans une moindre mesure, au Pérou. Comme le signale un rapport récent de la Commission Économique pour l'Amérique latine et les Caraïbes (CEPAL), l'inflation a reculé de manière manifeste et la situation externe de la région s'est améliorée, malgré un contexte international relativement défavorable ${ }^{2}$. Au-delà de ces chiffres, il convient de souligner l'importance que revêtent les processus d'ajustement et de reconversion économique entamés dans plusieurs pays latino-américains qui, en dépit de leurs caractères rigoureux et de leurs coûts sociaux incontestables, semblaient indispensables étant donné l'épuisement du modèle traditionnel de substitution des importations, qui s'est fait à l'abri de barrières protectionnistes et moyennant une intervention active de l'État. Bien que ce modèle ait produit des résultats assez intéressants pendant plusieurs décennies, il a atteint la limite de ses possibilités dans les années 70 .

3 De nombreux pays latino-américains tentent de dépasser l'épuisement de la précédente stratégie de développement et de la remplacer par des modèles d'économie de marché ouverts à l'extérieur. Les réformes économiques mettent l'accent sur la libéralisation commerciale, l'adoption de taux de change plus concurrentiels, l'encouragement aux investissements extérieurs, la libéralisation financière, une reconnaissance accrue du rôle du secteur privé et la privatisation de nombreuses entreprises publiques ${ }^{3}$. Les résultats de ces changements deviennent perceptibles, comme le démontrent les cas du Chili, du Mexique, de la Bolivie ou, plus récemment, de l'Argentine. Mais ce sont certainement des processus de conversion difficiles et douloureux, qui créent des tensions sociales et peuvent avoir des répercussions politiques, comme semble le montrer le cas vénézuélien, même s'il serait simpliste de n'attribuer la tentative de coup d'Etat de février 1992 qu'au programme d'ajustement économique entrepris par le gouvernement de Carlos Andrés Pérez.

4 A long terme, les perspectives économiques de l'Amérique latine ne paraissent pas si modestes. La région dispose d'un ensemble très favorable de ressources naturelles, comprenant un secteur agricole doté de puissants avantages comparatifs, temporairement annulés par le protectionnisme agricole européen, un secteur minier diversifié, et de considérables réserves pétrolières. Cette richesse est complétée par un secteur industriel assez important, qui continuera à s'insérer dans une économie globale de plus en plus interdépendante, basée sur la spécialisation géographique et l'existence de processus transnationaux de production. La région possède également des cadres 
techniques et administratifs de bon niveau qui, s'ils sont concentrés dans les pays de plus grand développement relatif, sont habitués à opérer avec flexibilité dans des milieux changeants, voire hostiles. Des marchés du travail plus flexibles et moins segmentés pourraient également contribuer à une réinsertion de la région dans l'économie internationale. Ces avantages relatifs, joints aux facilités prévues pour la conversion de la dette extérieure en actions d'entreprises locales et à la rentabilité élevée de divers instruments financiers latino-américains, ont permis un important retour des investissements étrangers directs et de portefeuilles, vers la région, une tendance à laquelle les investisseurs européens ont participé de manière notable. Le poids de la dette extérieure reste un problème grave pour de nombreux pays, mais la relation entre le service de la dette et les revenus d'exportation devient plus favorable. En 1991 également, il s'est produit une hausse considérable dans les cotisations de la dette latino-américaine sur le marché secondaire. Toutes ces tendances permettent un optimisme prudent quant à l'avenir économique de l'Amérique latine, qui apparaît dans des évaluations réalisées par des organes aussi prestigieux et influents que « The Economist $»^{4}$ et le « Time $»^{5}$.

Ce bilan favorable se voit d'une certaine manière confirmé par les nouvelles conditions politiques, internes et externes, qui se manifestent dans la région. Malgré les incontestables problèmes qui subsistent dans ce domaine, la démocratie n'a jamais été aussi répandue en Amérique latine. Il est certain que l'on enregistre encore de graves violations des droits de l'homme dans différents pays, que la violence politique persiste en Colombie et au Pérou. Il est vrai aussi que les processus de démocratisation ont avorté en Haïti et au Pérou, que, cette année (1992), l'une des démocraties les mieux assises de la région - le Venezuela - a dû affronter une émeute militaire, et que le régime cubain s'accroche à un modèle d'organisation politique et économique virtuellement abandonné dans le monde. Cependant, que l'on applique une perspective historique, ou que l'on compare la réalité de la région à celle d'autres régions en développement, les progrès réalisés paraissent considérables, même s'il reste beaucoup de chemin à parcourir.

6 Du point de vue international, la fin de la guerre froide a signifié la diminution d'une source de tension permanente pour la région. Le conflit centre-américain, tel que nous l'avons connu dans les années 80, appartient au passé. Même le conflit intérieur qui semblait le plus persistant et le plus insoluble, la cruelle guerre civile au Salvador, commence à se résoudre. Les pays latino-américains ont aussi tiré parti des puissants liens historiques, économiques, politiques et culturels qui les unissent, en développant de nouvelles formes de coopération dans des domaines très divers.

\section{Coopération et intégration régionale : une nouvelle impulsion}

7 Les relations entre les pays latino-américains ont été marquées traditionnellement par un certain dualisme. D'une part, elles sont caractérisées par un élan répétitif vers l'intégration et la coopération régionale, nourri par une histoire commune, la proximité géographique, des intérêts économiques, des affinités politiques et culturelles, des perceptions partagées quant au contexte extérieur de la région, et des idéaux d'unité régionale qui datent de la période de l'indépendance. D'autre part, les relations intrarégionales ont aussi été affectées par des conflits et des rivalités, attribuables à une longue histoire de disputes territoriales, de différends idéologiques, 
de pressions économiques et démographiques et, surtout, de méfiances et d'obsessions alimentées par de vieilles conceptions géopolitiques.

8 Il n'est pas douteux que l'élan vers l'intégration et la coopération régionale l'a emporté au cours des dernières années. Cette prépondérance s'est traduite par la multiplication d'initiatives et la création d'une série de mécanismes d'intégration et de coopération, couvrant des zones géographiques et des thèmes très variés. Les mécanismes peuvent être formels ou informels, multilatéraux ou bilatéraux, régionaux ou sous-régionaux, larges ou restreints. Les objectifs de ces actions et de ces schémas se réfèrent à des thèmes aussi divers que la libéralisation des échanges commerciaux, le rapprochement de marchés, le développement de l'infrastructure physique, l'utilisation de ressources communes, le développement technologique, la mise en route de systèmes de paiements pour le commerce régional, l'adoption de mesures de confiance mutuelle, l'établissement d'institutions de coopération politique régionale, la concertation en matière de politique extérieure, etc.

Comme le rappelle le Secrétaire exécutif de la CEPAL, les postulats de l'intégration économique en Amérique latine ont connu un important bouleversement par rapport à des périodes antérieures. $\mathrm{Si}$, dans le passé, l'intégration était conçue comme un instrument de défense collective contre les revers du secteur externe, elle a aujourd'hui tendance à être considérée comme un élément plutôt offensif, qui contribue à améliorer l'insertion internationale de l'Amérique latine ${ }^{6}$. Si, auparavant, l'accent était mis sur la protection des marchés, la régulation et la répartition des secteurs pour soutenir les industries naissantes des pays membres, et le commerce administré, il s'agit aujourd'hui d'accroître la compétitivité internationale et d'encourager la libéralisation conjointe. Si l'intégration représentait hier le corollaire logique du modèle de développement basé sur la substitution des importations, elle tente aujourd'hui de s'adapter aux nouvelles conceptions économiques qui s'imposent dans la région.

Bien que le schéma d'intégration géographiquement le plus étendu - l'Association LatinoAméricaine d'Intégration (ALADI) - ait enregistré peu de progrès, il a au moins servi à agencer une série d'initiatives sous-régionales qui semblent plus viables. Parmi celles-ci, on remarquera surtout le Marché Commun du Cône sud (Mercosur), une entité qui regroupe l'Argentine, le Brésil, le Paraguay et l'Uruguay; dans l'espoir de former un marché commun, fin 1994, et atteindre la libre circulation des biens, des services, des capitaux et de la main-d'oeuvre début 1996. Cette expérience à peine lancée et la situation de forte instabilité économique qui persiste au Brésil rendent difficile et prématurée l'évaluation de la viabilité de ce schéma. En définitive, son succès dépendra de la stabilisation économique des deux principaux associés, d'une meilleure harmonisation des politiques macro-économiques entre les pays membres, de l'instauration d'un appareil institutionnel qui semble encore très fragile, et du dépassement des réticences brésiliennes traditionnelles vis-à-vis de toute tentative de supranationalité.

11 Quoiqu'il en soit, le potentiel du Mercosur paraît très important, étant donné le poids économique et les niveaux de développement industriel, agricole et technologique de ses deux membres principaux. La consolidation de ce schéma pourrait mener à l'incorporation de nouveaux membres, parmi lesquels le Chili, qui a adopté une position d'expectative attentive face à ce processus d'intégration sous-régionale. Le cas de la Bolivie semble plus problématique, dans la mesure où, bien que son économie soit de plus en plus orientée vers les pays du Mercosur, elle est un des membres fondateurs du Groupe andin. 

de revitalisation, fort prometteur si l'on considère sa trajectoire antérieure et les niveaux d'interdépendance existant entre ses pays membres. Ce nouvel élan est alimenté par l'intense concertation politique que les pays de l'isthme centre-américain ont réalisée dans les années 80. Dans le cas du Groupe andin (Bolivie, Colombie, Équateur, Pérou et Venezuela), des initiatives successives de revitalisation ont été enregistrées au cours des dernières années, dans le but d'accélérer l'intégration entre les pays membres, à partir d'un modèle plus conforme aux nouvelles conceptions économiques en vigueur en Amérique latine. Même si les résultats finaux de ces efforts semblent encore incertains, ils ont entraîné l'adoption de programmes de libéralisation assez ambitieux, qui dépassent le cadre purement commercial. Deux membres du Groupe Andin - la Colombie et le Venezuela - font partie avec le Mexique de ce que l'on appelle le Groupe des Trois; il constitue une importante instance de coopération politique en Amérique latine, mais il s'est proposé également des objectifs à caractère économique, telle que la souscription d'un accord de libre-échange qui entrerait en vigueur en 1993.

13 Mais les schémas sous-régionaux n'épuisent pas les possibilités dans ce domaine. Le Mexique et le Chili ont signé, en 1991, un intéressant accord de complémentarité économique qui établit une zone de libre-échange entre les deux pays. Les pays d'Amérique centrale et le Mexique ont décidé, en janvier 1991, de créer une zone de libreéchange qui devrait être opérationnelle fin 1996. Le Venezuela a proposé le libre accès des produits centre-américains à son marché, sans exiger la réciprocité. L'Argentine et le Chili ont négocié d'importants accords pour promouvoir l'intégration physique entre leurs pays. La Colombie, le Mexique et le Venezuela se sont engagés à créer leur propre zone de libre-échange. Les compatibilités entre ces initiatives et leurs résultats concrets sont encore incertains, mais il est évident qu'elles traduisent un nouveau climat de coopération dans la région, basé davantage sur des intérêts pragmatiques que sur les anciennes utopies d'unité régionale.

tes années 80, un développement remarquable a été enregistré dans la coopération politique régionale en Amérique latine, particulièrement en ce qui concerne le conflit centre-américain. Les nouvelles modalités de coopération créées dans ce contexte ont conduit à la constitution du Groupe de Contadora, puis à son Groupe d'appui, qui ont joué un rôle essentiel dans la recherche d'une solution pacifique pour le conflit centre-américain, rendue possible grâce à une action collective sans précédent dans la région ${ }^{7}$. A partir de 1986, les principales initiatives de médiation dans le conflit centre-américain provenaient des pays impliqués, et ont été canalisées dans le processus de paix d'Esquipulas qui a ouvert une nouvelle étape de concertation politique en Amérique centrale ${ }^{8}$. L'expérience de ces années fut essentielle pour le développement de la coopération politique régionale en Amérique latine car, pour la première fois depuis longtemps, un ensemble de pays de la région, qui affichaient des positions très différentes, se concertaient pour trouver une solution adéquate à un conflit extérieur majeur.

15 L'expérience accumulée dans le cadre du conflit centre-américain a puissamment influé sur la mise en place, en 1986, du mécanisme permanent de consultation et de coordination, plus connu sous le nom de Groupe de Rio. Il compte actuellement onze membres à part entière - le Mexique et tous les pays sud-américains de souche ibérique et deux observateurs, qui représentent respectivement les pays de l'isthme centreaméricains et ceux des Caraïbes. Le groupe est devenu progressivement la plus 
importante instance de coopération politique et de dialogue politique extérieur dans la région'. Cette dernière dimension s'est révélée particulièrement importante dans le dialogue politique Europe-Amérique latine, puisque, depuis 1987, des réunions périodiques se tiennent au niveau ministériel entre les pays du Groupe de Rio et leurs collègues des pays membres de la Communauté européenne (CE). Comme toute expérience de coopération politique régionale, ce processus n'est pas exempt de frustrations et est handicapé par des excès de rhétorique et de volontarisme qui donnent parfois l'impression (fausse) que le Groupe de Rio est une instance d'intégration économique, voire d'unification politique, des objectifs qui semblent entre très lointains et ne correspondent pas à un processus de cette nature. Néanmoins, le bilan de cette expérience de coopération est plutôt favorable.

$\mathrm{Au}$ cours des dernières années, la coopération politique régionale s'est occupée particulièrement de la défense et de la promotion de la démocratie en Amérique latine, surtout des cas de Haïti, de Cuba et, plus récemment, du Pérou. Les trois cas illustrent le dilemme qui se pose entre deux principes de base adoptés en Amérique latine: d'une part, la non-intervention et, de l'autre, la défense de la démocratie et des Droits de l'homme. Les pays latino-américains ont adopté, face à ce dilemme, des positions diverses. Au cours des dernières années du moins, le Venezuela et l'Argentine se sont montrés partisans d'une action énergique dans la défense et la promotion de la démocratie, tandis que le Mexique et le Brésil ont maintenu des positions plus prudentes, dans le but de préserver le principe de non- intervention et de ne pas créer de précédents qui pourraient avoir des conséquences délicates et dangereuses. En fin de compte. le consensus régional s'est situé entre ces deux positions extrêmes et, dans les faits, on a adopté une optique de cas par cas. Le régime militaire de Haïti a été l'objet de sanctions et d'un embargo commercial, décrété dans le cadre de l'Organisation des États américains (OEA), qui n'a pas été suivi avec la même intensité par les pays de la CE. Dans le cas du Pérou, on a préféré maintenir sur le plan diplomatique la pression pour le rétablissement de la démocratie. Quant à Cuba, les pays du Groupe de Rio ont utilisé davantage la persuasion pour que l'île atteigne dans la paix « la justice, la liberté, la démocratie, le respect des droits de l'homme et un développement économique ouvert et libre », afin d'obtenir la tant souhaitée réintégration de Cuba dans le système interaméricain ${ }^{10}$.

17 Les pays latino-américains déploient de gros efforts pour mettre fin aux anciennes rivalités et aux lointains conflits. La coopération entre l'Argentine et le Brésil déborde le domaine purement économique et a impliqué un changement profond dans des relations bilatérales parfois marquées par la rivalité et la méfiance mutuelles ${ }^{11}$.

L'Argentine, le Brésil et le Chili ont souscrit à Mendoza un accord important qui interdit les armes de destruction massive. L'Argentine et le Chili ont signé en 1991 un accord formel visant à résoudre tous les différends frontaliers encore en suspens, y compris un traité d'arbitrage pour le cas le plus complexe. La Colombie et le Venezuela entretiennent des pourparlers depuis 1990 afin de résoudre leur différend maritime. Début 1992, le Pérou a octroyé certaines facilités pour alléger les problèmes dérivant du caractère méditerranéen de la Bolivie. Bien que les relations diplomatiques formelles soient toujours interrompues entre la Bolivie et le Chili, l'essor du commerce bilatéral a mené à la négociation d'un accord économique entre les deux pays. La visite historique effectuée, début 1992, par le président péruvien Fujimori en Équateur a permis de faire baisser la traditionnelle tension frontalière entre les deux pays. Il serait naif de conclure que les vieilles obsessions géopolitiques ont disparu de la région ou que toutes les hypothèses de 
conflit ont été surmontées. Cependant, le développement de la démocratie et une conscience croissante des réalités et des exigences de l'interdépendance ont créé un climat nouveau dans les relations entre pays latino-américains, dont l'effet le plus important a été une lente réduction des dépenses militaires.

\section{Les relations extérieures de l'Amérique Latine : la montée du pragmatisme}

Les relations extérieures de la région se sont structurées, au cours des dernières décennies, autour de trois axes principaux: États-Unis, Europe et bassin du Pacifique.

Les États-Unismaintiennent dans la région une présence prioritaire comme principal partenaire commercial et financier, comme source d'investissements et, pour l'Amérique centrale et les Caraïbes, en tant que source vitale de coopération et d'aide militaire, et en tant qu'acteur politique d'une importance indéniable. Bien qu'il existe une asymétrie évidente dans les relations interaméricaines, l'Amérique latine est plus intéressante pour les États-unis que pour ses autres partenaires extérieurs comme marché de destination pour leurs exportations et leurs investissements, comme source d'approvisionnement en matières premières, comme partenaire industriel dans des processus de production de plus en plus transnationalisés, et comme partenaire financier souvent problématique. L'Amérique latine représente aussi un intérêt stratégique pour Washington. Le bassin des Caraïbes a été défini dans le périmètre essentiel de sécurité de la grande puissance. Le Conseil de sécurité nationale nord-américain traite régulièrement les problèmes qu'il relève dans les îles des Caraïbes, au Mexique et en Amérique centrale. L'intérêt des Étatsunis pour révolution politique de l'Amérique latine a été démontré, parfois en termes tragiques, en de multiples occasions, et représente aujourd'hui un point de coïncidence avec les nouveaux régimes démocratiques de la région ${ }^{12}$.

Les notions anciennes, souvent simplistes, sur la domination impérialiste des États-unis en Amérique latine ne sont jamais parvenues à couvrir la complexité et la diversité de l'évolution historique de ces relations. Celles-ci ont suivi des voies très distinctes, par exemple en Amérique centrale ou dans le Cône sud, et qui ont accusé l'impact des changements importants enregistrés tant aux États-Unis que dans les pays latinoaméricains. Même si la puissance nord-américaine est toujours l'acteur extérieur qui a le plus de poids dans la région, sa présence n'est pas aussi universelle qu'on le croit. Des pays importants de l'Amérique latine, comme le Brésil, l'Argentine, le Chili ou la Colombie, commercent davantage avec la CE qu'avec les États-Unis. La banque japonaise a supplanté ces dernières années celle des États-Unis comme principal bailleur de fonds pour les pays latino-américains ${ }^{13}$. Le rôle décroissant des États-Unis comme exportateur de capitaux a amené l'Europe et le Japon à remplacer la grande puissance en tant que source d'investissements nouveaux dans la région. Les sérieuses limitations législatives qui touchent l'aide au développement aux États-Unis ont empêché l'exécution des promesses relativement discrètes formulées suite aux changements de régime à Panama et au Nicaragua. Il est certain que ces réductions peuvent avoir un caractère conjoncturel et se produisent à partir de niveaux préalables particulièrement élevés, mais il est tout aussi vrai que les relations économiques de l'Amérique latine se sont diversifiées de manière appréciable dans les dernières décennies. 
22 La situation n'est pas très différente dans le domaine politique. Le conflit centreaméricain a démontré que même les pays les plus vulnérables à l'influence nordaméricaine ont développé une certaine capacité à définir leurs propres intérêts externes et internes. Cette capacité s'est reflétée aussi bien dans la mise sur pied du processus de paix régionale que dans la recherche d'autres partenaires extérieurs, y compris la CE et ses pays membres. La plus grande autonomie relative n'est pas toujours positive: l'interruption des relations militaires entre les États-Unis et le Guatemala depuis les années 70 a privé Washington d'un instrument de pression sur les forces armées guatémaltèques pour un plus grand respect des Droits de l'homme. Quant aux plus grands pays d'Amérique latine, les États-Unis n'ont pu exercer une influence décisive sur leur évolution politique interne que dans des cas tout à fait exceptionnels.

Les thèmes les plus polémiques des relations entre les États-Unis et l'Amérique latine se sont déplacés principalement vers le domaine économique. Les préoccupations commerciales commencent à prendre le pas sur les anciens conflits politiques ou la traditionnelle méfiance latino-américaine vis-à-vis de l'hégémonie nord-américaine. L'invasion de Panama en 1990 a affaibli un principe de base des relations interaméricaines, mais ses répercussions dans la région ont été très limitées. Par contre, les différends commerciaux du Brésil ou du Chili avec les États-Unis reçoivent généralement une attention prioritaire dans les milieux politiques et l'opinion publique de ces pays.

C'est dans ce contexte que s'explique l'intérêt qu'a éveillé en Amérique latine l'Initiative pour les Amériques, annoncée par le président Bush en juin 1990. La proposition nordaméricaine vise à promouvoir la libéralisation du commerce entre les États-Unis et les pays latino-américains, dans le but d'établir une zone de libre-échange qui engloberait tout le continent, de réduire la dette contractée par les pays latino-américains vis-à-vis du gouvernement nord-américain et de stimuler les investissements en Amérique latine. Il est également prévu de mettre en oeuvre des schémas de conversion de la dette en programmes de coopération pour la protection de l'environnement ${ }^{14} \mathrm{La}$ mise en route de l'Initiative a été progressive, parce qu'elle dépend en grande partie de l'approbation d'un « paquet » législatif aux États-Unis. Toutefois, il est peu probable qu'elle réponde aux grandes expectatives qu'elle a éveillées dans de nombreux pays latino-américains. De fait, même si plusieurs pays de la région se montrent très désireux de s'intégrer à une zone de libre-échange, et que certains analystes aient prédit la constitution virtuelle d'un bloc commercial américain, la viabilité de cette idée paraît limitée, surtout en raison des tendances protectionnistes des États-Unis. Ainsi, l'insertion extérieure de l'Amérique latine ne tourne pas exclusivement autour de cette possibilité. Les progrès de la négociation pour l'adhésion du Mexique à l'Accord nord-américain de libre-échange (NAFTA), comprenant les États-Unis et le Canada, répondent à un processus distinct et antérieur à l'Initiative pour les Amériques, qui vise en réalité à réguler formellement une relation d'étroite interdépendance économique entre les États-Unis et le Mexique, mais qui ne s'étend pas aux économies d'Amérique du Sud.

L'Amérique latine acquiert également une importance croissante liée à certains nouveaux intérêts de sécurité des États-Unis. Parmi ceux-ci, on remarquera des thèmes complexes, tels que les migrations en provenance du Mexique, des Caraïbes et de l'Amérique centrale, la protection de l'environnement et le trafic de drogue ${ }^{15}$. La libéralisation commerciale a été liée au problème des migrations, se basant sur l'argument que l'amélioration des conditions économiques dans les pays d'origine pourrait réduire l'effet d'attraction 
du marché nord-américain, mais l'expérience de Porto Rico oblige à nuancer cet argument. La protection de l'environnement mène à l'application de nouvelles conditions dans les relations de l'Amérique latine avec les États-Unis (et avec d'autres partenaires extérieurs). L'objectif du contrôle du trafic de drogue, défini comme une menace pour la sécurité nationale des États-Unis, a suscité de vastes programmes de coopération économique et policière, non exempts de polémique dans le dernier cas.

Au début des années 90, l'Europe est toujours perçue comme un partenaire considérable pour l'Amérique latine, malgré la place incontestablement modeste qu'occupe la région dans les priorités économiques et politiques européennes. La CE conserve solidement sa position de deuxième partenaire commercial de l'Amérique latine. Les pays membres de la CE représentent actuellement la principale source de coopération disponible pour la région. Le rôle de l'Europe comme fournisseur d'investissements directs et indirects en Amérique latine s'est accru au cours des dernières années. Il ne faut pas non plus sousestimer le poids des créanciers européens dans la dette extérieure latino-américaine, dépassés seulement par ceux des États-Unis, ni l'influence nettement orthodoxe des cercles financiers et gouvernementaux européens sur les positions qui sont adoptées dans ce domaine ${ }^{16}$.

Les principaux problèmes des relations Europe-Amérique latine se situent au niveau commercial et sont dus essentiellement aux barrières non-tarifaires qui limitent le commerce avec l'Amérique latine dans des secteurs très sensibles, les secteurs agricole, textile et sidérurgique ${ }^{17}$. L'espoir de surmonter certains de ces problèmes dépend de l'issue de l'Uruguay Round, car des négociations directes entre la CE et l'Amérique latine ne semblent pas viables à cet égard. La participation décroissante de l'Amérique latine dans le commerce extérieur de la CE n'est pas due uniquement au protectionnisme européen, elle reflète également le problème de la perte d'importance de nombreux produits latino-américains traditionnels, ainsi que le manque de compétitivité des exportations latino-américaines à plus grande valeur ajoutée, surtout par comparaison avec le Sud-Est asiatique. ${ }^{18}$

28 La présence économique européenne en Amérique latine est assez diversifiée. La présence économique allemande est manifeste dans le domaine commercial, la coopération au développement et les investissements. Le Royaume-Uni garde une position importante dans le domaine financier et comme source d'investissements, provenant en partie des schémas de conversion de la dette en actions. L'Italie a développé de manière significative ses programmes de coopération dans la région et a usé des accords bilatéraux souscrits avec des pays comme l'Argentine, le Venezuela, le Brésil ou le Chili. La France reste un partenaire commercial important dans la région et se spécialise également dans la construction de grands travaux publics. L'Espagne a souscrit des accords de coopération et d'amitié avec presque tous les pays économiquement plus avancés de la région afin d'y stimuler ses investissements et de promouvoir la création d'entreprises conjointes. Les Pays-Bas représentent la troisième source de coopération au développement en Amérique latine et restent un acheteur relativement important de produits de base latinoaméricains.

Les relations politiques entre les pays européens et latino-américains ont pris un essor important ces dernières années. En 1987, un dialogue politique interrégional a été entamé par les pays membres de la $\mathrm{CE}$, dans le cadre de la coopération politique européenne, avec 
les pays du Groupe de Rio. L'institutionnalisation de ce dialogue, fin 1990, a répondu à une aspiration latino-américaine. Bien que ce forum soit à caractère politique, les pays latinoaméricains l'utilisent pour débattre des problèmes de la coopération économique entre les deux régions, compensant ainsi l'absence de mécanismes efficaces de dialogue dans ce domaine.

Les pays et les institutions communautaires européennes se sont également engagés visà-vis de la pacification, la démocratisation et le développement de l'Amérique centrale. Dans le domaine économique, cet engagement s'est traduit par un programme intéressant de coopération avec les pays de l'Isthme ${ }^{19}$. Au niveau politique, l'Europe a joué un rôle actif de soutien au processus de paix centre-américain dans les années 80 . De même, plusieurs pays européens, et particulièrement l'Espagne, ont contribué aux efforts de médiation pour mettre fin aux conflits internes dans les pays centre-américains et se sont joints aux forces des Nations Unies qui supervisent les processus de paix.

31 Si les relations de l'Amérique latine avec l'Europe occidentale présentent un profil stable, celles qu'elle entretient avec les États successeurs de l'Union Soviétique et avec les pays d'Europe centrale et de l'Est traversent une période d'incertitude et de redéfinition, conséquence des profonds changements qui ont suivi l'effondrement des régimes communistes. Cuba est le pays latino-américain le plus touché par ces changements, en raison de son extrême dépendance de la coopération soviétique ${ }^{20}$. L'île subit une crise économique extrêmement grave, mais le gouvernement de Fidel Castro s'accroche à un modèle économique et politique qui a échoué dans le reste du monde. Ni l'Amérique latine ni l'Europe ne prétendent contribuer à l'implacable blocus économique que les Etats-Unis appliquent à Cuba depuis trois décennies, mais elles ne sont pas non plus disposées à remplacer l'ancienne Union Soviétique dans son rôle de bienfaitrice du régime cubain. Quant au reste de l'Amérique latine, l'impact des changements touche certains courants commerciaux significatifs, surtout dans le cas de pays comme l'Argentine et le Brésil ${ }^{21}$. A long terme, des perspectives intéressantes peuvent s'ouvrir dans les relations de l'Amérique latine avec la Russie ou certains pays d'Europe centrale et de l'Est, mais pas au point de recréer un axe particulier des relations extérieures de l'Amérique latine.

32 Par contre, les relations de la région avec les pays du bassin du Pacifique ont connu une croissance progressive mais soutenue au cours des dernières années. Le Japon fournit près de $7 \%$ des importations et reçoit environ $5 \%$ des exportations latino-américaines, essentiellement dans le cas de pays comme le Chili, le Mexique, le Pérou ou Panama. La banque japonaise a acquis une présence financière importante dans la région. De plus en plus d'entreprises japonaises effectuent de gros investissements en Amérique latine. Le Japon est aussi devenu le pays qui offre le plus de coopération au développement en Amérique latine. Ces données ne reflètent pas nécessairement une priorité de la part de Tokyo, puisqu'au cours des dernières années, on a remarqué une attitude japonaise plutôt prudente vis-à-vis de la région ${ }^{22}$. Il s'agit davantage d'une preuve supplémentaire de la montée du Japon comme puissance économique mondiale.

La présence japonaise en Amérique latine ne s'est pas encore traduite sur le plan politique. Bien que Tokyo ait tenté d'adopter des positions propres face à certains thèmes latino-américains, elle préfère maintenir un profil politique bas, suivant une tendance générale de sa politique extérieure, à cette différence près que, dans le cas latinoaméricain, il semble encore plus enclin à croire que l'initiative doit dépendre principalement de son allié nord-américain ${ }^{23}$. Soit dit en passant, cette attitude de profil 
politique bas contraste avec la position européenne, bien plus engagée avec la cause de la démocratisation et d'autres thèmes politiques dans la région.

Les pays latino-américains ont tenté d'accroître leur présence dans le bassin du Pacifique en intensifiant leurs relations avec le Japon, en normalisant ou en élargissant leurs relations avec la République populaire de Chine, et en développant des liens économiques avec la Corée du Sud, Taiwan, les pays membres de l'ASEAN, l'Australie et la NouvelleZélande. Le Chili, le Mexique et le Pérou, qui semblent les plus intéressés par une ouverture vers le Pacifique, ont également adhéré à divers mécanismes de coopération dans le bassin du Pacifique, même si toutes les réserves n'ont pas encore été levées pour une participation totale à ce niveau ${ }^{24}$

Comme on le voit, les relations internationales de l'Amérique latine semblent plus diversifiées et plus riches que ce que suggèrent les analyses conventionnelles sur l'hégémonie nord-américaine dans la région. S'il est manifeste que la présence des ÉtatsUnis reste importante, elle n'a pas exclu l'établissement de liens intéressants et d'un grand potentiel entre les pays latino-américains eux-mêmes, et entre ceux-ci et les autres acteurs principaux sur la scène internationale.

La région ne paraît pas non plus si mal préparée pour affronter les nouveaux défis que pose un système international en transition. Il est certain que la région est handicapée par de gros problèmes économiques et sociaux, mais il est tout aussi vrai que des indices permettent d'envisager une importante récupération économique, que des succès notoires ont été remportés dans le domaine politique, surtout en comparaison avec la situation désolante enregistrée dans les années 70 , et que de nouvelles voies s'ouvrent pour la coopération régionale, guidées par un réalisme salutaire.

En définitive, l'exploitation de ces tendances favorables dépendra des latino-américains eux-mêmes. La coopération internationale peut certainement jouer un rôle intéressant de soutien, mais elle ne peut ni ne doit remplacer les efforts à déployer dans la région pour moderniser les économies, améliorer les conditions sociales qui sont souvent profondément anachroniques, et consolider les institutions politiques démocratiques.

\section{NOTES}

1. Voir par exemple son intervention à un séminaire qui s'est tenu à Oslo, El Pais, 9/12/1991.

2. CEPAL, «Balance Preliminar de la Economia de América Latina y El Caribe », Santiago du Chili, Doc. LC/G.1696, 18 décembre 1991.

3. Voir sur ce thème John Williams on , The Progress of Policy Reform in Latin America, Institute for International Economics, Washington D.C., 1990.

4. $19 / 10 / 1991$

5. $28 / 10 / 1991$

6. Gert Rosenthal, «Un informe critico a 30 anos de integracion de América Latina ", Nueva Sociedad, 113, mai-juin 1991, pp. 60-66.

7. Nous avons traité ce sujet dans Alberto van Klaveren, «Las nuevas formas de concertacion politica en América Latina », Estudios Internacionales, 68, octobre-décembre 1984, pp. 513-536. Voir 
également Luciano Tomassini (comp.), Nuevas formas de cooperacion regional en América Latina, Buenos Aires, Grupo Editor Latinoamericano, 1990.

8. Voir sur ce thème Francisco Rojas Aravena, «El proceso de Esquipulas: el desarrollo conceptual y los mecanismos operativos ", Sintesis, 7 janvier-avril 1989, pp. 208-225.

9. Voir sur ce thème Alicia Frohman, Puentes sobre la turbulencia. La concertacion politica regional en América Latina, Santiago du Chili, FLACSO, 1990 ; IRELA, El Grupo de los Ocho: Un nuevo interlocutor regional en América Latina?, Madrid, Dossiers d'IRELA, 1989 et Tomassini, Nuevas fonnas de concertacion, op.cit.

10. El Pais, 02/12/1991.

11. Nous avons traité ce thème de manière plus détaillée dans Alberto van Klaveren, «Democratizacion y politica exterior : el acercamiento entre Argentina y Brasil », Revista CIDOB d'Afers Intemacionals, 18, 1990, pp. 13-44.

12. Voir à ce sujet les travaux d'Abraham Lowenthal (ed.), Exporting Democracy. TheUnited States and Latin America, 2 vols, The John Hopkins University Press, Baltimore, 1991 et Thomas Carothers, In the Name of Democracy. U.S. Policy Toward Latin America in the Reagan Years, University of California Press, Berkeley, 1991.

13. Barbara Stallings et G. Szekely, «A Raising Sun in Latin America », Hemisfile, mars 1990.

14. Sur l'Initiative des Amériques, voir le numéro de Capitulos del SELA, 28, 1991, consacré à «L'analyse régionale de l'Initiative des Amériques ».

15. Lowenthal Abraham, Partners in conflict. The United States and Latin América, The John Hopkins University Press, Baltimore, 1987, pp .48-65.

16. On trouvera un bilan plus détaillé des relations Europe-Amérique latine dans Jorge Grandi et al., Les relations Europe-Amérique latine et le Marché Unique Européen, Cahiers CERCAL, n7, Bruxelles, 1992.

17. CEPAL, «Las barreras non arancelarias a las exportaciones latinoamericanas en la Comunidad Europea ", document préparé par la Direction du Commerce International et Développement (miméo), 1990.

18. Kees den Boer, «El estado actual y las perspectivas de las relaciones comerciales y economicas entre Europa (la CE) y América Latina ", publié in : Divers auteurs, La nueva Europa y el futuro de América Latina, Ajuntament de Barcelona/instituto de Cooperacion Iberoamericana, Barcelona, 1990, pp. 203-216.

19. José Roberto Lopez, Las relaciones economicas entre la Comunidad Europea y América Central durante los anos ochenta : balance y perspectivas, Documento de Trabajo del IRELA, $\mathrm{n}^{\circ} 24$, Madrid, 1990.

20. Carmelo Mesa-Lago et al., «Relaciones economicas de Cuba con la URSS y el CAME : pasado, presente y futuro ", communication présentée à la seconde réunion du Groupe d'Etude de LASA sur «América Latina, Cuba y la Economia Internacional», Centro de Investigaciones de la Economia Mundial, La Havane, 9-10 juillet 1990 et Jorge Pérez-Lopez, « Nadando contra corriente : Implicaciones para Cuba de las reformas en las relaciones economicas internacionales soviéticas y de los paises de Europa del Este ", Sintesis, n¹5, Madrid, 1991, pp. 299-346.

21. Aldo Vacs, "Crisis, Reform, and Accomodation: the Impact of Soviet Perestroika on Argentina and Brazil ", communication présentée au XVI ${ }^{\mathrm{e}}$ congrès international de la Latin American Studies Association (LASA), Washington D.C., du 4 au 6 avril 1991.

22. Barbara Stallings et Kotaro Horisaka, «Japanese Relations with Latin America : Looking Towards the 1990s ", communication présentée à la conférence «Latin America and the United States in a New World», organisée par Dialogo Interamericano, Aspen Institute, Washington D.C., 13-14 juin 1991.

23. Hiroshi Matsushita, «La politica japonesa hacia América Latina en la década de 1980. Discrepancia y colaboracion con los Estados Unidos ", communication présentée au XV $\mathrm{XV}^{\mathrm{e}}$ congrès international de LASA, Miami, du 3 au 6 décembre 1990. 
24. Francisco Orrego Vicuna, «La cooperacion en el Pacifico : una perspectiva desde América Latina », Estudios Internacionales, 22 : 86, Santiago, avril-juin 1989, pp. 150-151.

\section{RÉSUMÉS}

The author analyses the economic and institutional factors that enable to denote a certain recovery of most Latin-American countries' economic growth, a considerable consolidation of the democratic regimes in the region, although there are some important exceptions, the development of political cooperation between the most important countries, the settlement of bilateral or subregional tensions and the revival of regional integration processes. He proposes a detailed analysis of Latin America's complex and diversifted external relations, where the United States certainly do maintain a privileged position that they tried to consolidate with the Initiative for the Americas, but where Europe is also considered as an essential partner, even if economic relations have not followed the level of progress observed in the political field. The growing relations with countries of the Pacifie region, and mostly, the consolidation of intraregional cooperation, confirms this pragmatic approach of the region's future in the international scene.

\section{AUTEUR}

\section{ALBERTO VAN KLAVEREN}

Association de recherche et de spécialisation sur des thèmes ibéro-américains, Institut universitaire Ortega y Gasset, Madrid 